\title{
Evaluating Non-Linear Beamforming in a 3GPP-Compliant Framework Using the SWORD Platform
}

\author{
Marcin Filo, J.C. De Luna Ducoing, Chathura Jayawardena, Christopher Husmann, \\ Rahim Tafazolli, and Konstantinos Nikitopoulos \\ Wireless Systems Lab, 5G Innovation Centre, \\ Institute for Communication Systems, University of Surrey, \\ Guildford GU2 7XH, UK
}

\begin{abstract}
It is well documented that the achievable throughput of MIMO systems that employ linear beamforming can significantly degrade when the number of concurrently transmitted information streams approaches the number of base-station antennas. To increase the number of the supported streams, and therefore, to increase the achievable net throughput, non-linear beamforming techniques have been proposed. These beamforming approaches are typically evaluated via simulations or via simplified over-the-air experiments that are sufficient for validating their basic principles, but they neither provide insights about potential practical challenges when trying to adopt such approaches in a standards-compliant framework, nor they provide any indication about the achievable performance when they are part of a standards-compliant protocol stack. In this work, for first time, we evaluate non-linear beamforming in a 3GPP standardscompliant framework, using our recently-proposed SWORD research platform. SWORD is a flexible, open for research, softwaredriven platform that enables the rapid evaluation of advanced algorithms without extensive hardware optimizations that can prevent promising algorithms from being evaluated in a standardscompliant stack. We show that in an indoor environment, vector perturbation-based non-linear beamforming can provide up to 46\% throughput gains compared to linear approaches for $4 \times 4$ MIMO systems, while it can still provide gains of nearly $10 \%$ even if the number of base-station antennas is doubled.
\end{abstract}

\section{INTRODUCTION}

Non-linear beamforming/precoding has been proposed as an efficient method to improve the performance of the traditional, linearly-precoded MIMO systems [1]-[6]. Still, the evaluation of non-linear precoders has mainly been based either on linklevel simulations, or on simplified over-the-air (OTA) experiments where the corresponding processing takes place offline [7]. Such evaluation approaches can efficiently validate the proposed concepts, but are not adequate to identify and resolve issues related to the adoption of new algorithmic approaches by actual wireless standards, or to evaluate any actual performance gains, or drawbacks, when the full protocol stack (with the corresponding standards-compliant signalling) is involved. To identify and resolve such challenges, as well as to evaluate the actual gains of advanced algorithmic approaches in mobile systems, it is necessary to realize them in a full-stack, 3rd

978-1-7281-4490-0/20/\$31.00 @ 2020 IEEE
Generation Partnership Project (3GPP) standards-compliant environment.

In this direction, an appropriate research framework is required that would allow not only for the evaluation of ideas in a full-stack, standards-compliant setting, but also for the investigation of computationally-intensive approaches without any time-consuming hardware development. This last aspect is of particular importance in the case of non-linear precoders, where the computational complexity is high.

There is a number of both commercial and open-source platforms that can be used for conducting research and prototyping of new physical-layer approaches in a standards-compliant environment (e.g. OpenAirInterface (OAI) [8], srsLTE [9], openLTE [10], LTE Application framework [11], Amarisoft [12]). Still, the capabilities of these platforms are currently limited either to basic transmission modes (e.g., single-antenna transmission), or to very low-order multiple-input multipleoutput (MIMO) dimensions. In addition, in order to conduct OTA tests, tight real-time (RT) requirements need to be met, which limit the approaches that can be tested to only those with very low complexity. Consequently, existing platforms are not appropriate for the evaluation of non-linear precoding approaches. In order to circumvent these limitations, in this work we employ and extend our recently-proposed SoftWare Open Radio Design (SWORD) platform. SWORD supports large/massive MIMO setups in a 3GPP standards-compliant framework, as well as a new "pseudo-RT" operation mode that enables the rapid realization and OTA testing of highlycomplex algorithms [13]. As a part of SWORD we also proposed a digital signal processing (DSP) acceleration framework which reduces the execution time of computationally expensive MIMO operations by up to $88 \%$ [14]. Our evaluations presented here focus on an indoor transmission environment. This is not only because it is more practical in terms of obtaining measurements, but also since such indoor environments can substantially vary in terms of the MIMO channel condition (as we show in detail). Therefore, it allows us to relate the performance of non-linear precoding with specific transmission cases. In particular, we characterize the transmission channel in 
each measured location and discuss its impact on the gains of non-linear beamforming. We show that the channel condition can be a good (but not conclusive) indicator of the level of gains that can be achieved. This is highlighted by gains of up to $46 \%$ of non-linear beamforming when compared to a linear approach. To the best of our knowledge, this is the first time that a non-linear technique for multi-user (MU)-MIMO precoder is investigated in a full-stack, 3GPP standards-compliant setting, and related to specific transmission conditions.

\section{NON-LINEAR PRECODING}

Most existing MU-MIMO systems and research platforms employ linear processing for downlink beamforming, e.g., [15][20]. However, it is known that linear precoding techniques can provide satisfactory performance only when the transmission conditions are favorable (e.g., when the number of concurrently transmitted information streams is significantly smaller than the number of base-station antennas). To create an advantageous transmission condition, current MIMO testbeds are equipped with large numbers of antennas (e.g., tens or even hundreds) and support only a small number of users [17], [18], [21], which leaves on the table a lot of unexploited MIMO capacity. Still, as we show in Section V, even when the number of transmitted streams is much lower than the base-station antennas, the transmission condition can be unfavorable to linear precoding methods.

Employing optimal, non-linear processing (e.g., dirty paper coding-based techniques [1], [22]) can significantly increase the achievable throughput of MIMO systems [1]. In this direction, vector perturbation precoding can bridge the gap to the capacity limit, currently left unexploited by linear [17] and even sub-optimal non-linear [23] precoding techniques. In particular, non-linear precoders [1]-[6] perturb the constellation data symbols to maximize the received SNR. However, the latency and/or complexity of algorithms-such as sphere encodingthat calculate the perturbations are variable and can become impractical for large MU-MIMO systems. Therefore, most of the existing work in literature involving vector perturbation precoding are limited to link-level simulations [1]-[6].

The high latency and/or complexity requirements of traditional vector perturbation precoding combined with the saturating speed of processors, motivates a timely requirement for massively parallel processing [24]. In this work we adopt the recently-proposed massively parallel vector perturbation precoder of [7], which extends from our recently proposed generalized framework for massively parallel processing [25], [26], that can substantially reduce and fix the latency and/or complexity requirements of vector perturbation precoding. In particular, the adopted precoder can approach the throughput achieved by sphere encoding approaches with orders of magnitude lower complexity and with latency requirements similar to that of linear precoding [7].

It is interesting to note that none of the evaluations of the above approaches consider the impact of higher layers of the protocol stack, practical transmission rates, or other implementation challenges that affect the system-level throughput in a standards-compliant environment. In addition, since all the above approaches are highly-complex, a real-time evaluation of these in a 3GPP-compliant framework would require a substantial hardware implementation effort.

\section{NON-LINEAR EVALUATION VIA SWORD PLATFORM}

As discussed previously, having a proper research platform is crucial for boosting innovation and accelerating the development of novel physical-layer approaches. This is necessary not only to enable the evaluation of new physical-layer solutions in more realistic transmission scenarios, but also to permit researchers to rapidly identify and resolve any practical issues related with the use of such techniques in the actual wireless standards.

An important aspect of such a platform, as previously mentioned, is the capability for evaluating new approaches without the need for meeting strict timing requirements related with the RT execution. This is particularly important for the investigation of novel, computationally-intensive physical-layer techniques, such as non-linear beamforming. Traditionally, to circumvent the requirements related to the RT execution, researchers turn to non-RT processing in which a pre-generated signal is transmitted, sampled and then stored in a raw format on the receiver side for offline processing [7], [27], [28]. However, as this approach fails to capture dependencies between consecutive events (e.g. an erroneous reception is not followed by a retransmission), it is not suitable in the context of systemlevel evaluations with a full-protocol stack. Alternatively, a less commonly-used approach in which a pause period is introduced between the processing of consecutive transmission periods can be used instead. To the best of our knowledge, such a feature is not present in pre-existing research platforms.

Another important aspect of a research platform which is suitable for the investigation of non-linear beamforming is its capability to support large/massive MIMO setups. Although possible solutions exist (see e.g. LuMaMi [19], RENEW [29]), they are not designed to be used for evaluations as a part of a full 3GPP-compliant protocol stack. We note that there are also several research platforms which are specifically designed for the investigation and prototyping in a 3GPP standardscompliant environment (e.g. OAI [8], srsLTE [9], openLTE [10], or LabView based LTE Application Framework [11]). Still, as already discussed, these solutions do not yet provide support for large/massive MIMO setups.

In order to address the above shortcomings, here we employ our recently-proposed SWORD platform which is capable of supporting large/massive MIMO setups, with a complete 3GPP Long-Term Evolution (LTE)-compliant protocol stack implemented in software [13]. SWORD was specifically designed for rapid development, profiling, validation and testing of new approaches for next-generation networks. It was built around OAI which is currently the only software solution (except for a closed source solution by Amarisoft [12]) that provides an implementation of a full 3GPP protocol stack. In addition, OAI supports time-division duplexing (TDD) operation, which is suitable for large/massive MIMO systems. Furthermore, 
contrary to commercial solutions (e.g. LabView based LTE Application Framework [11]), it is freely available.

In order to use SWORD for the investigation of non-linear beamforming, we developed a number of extensions compared to the first iteration of the platform described in [13]. The first extension includes changes in the Radio Resource Control (RRC) signaling to indicate the use of non-linear beamforming to user equipment devices (UEs). This was necessary to indicate the use of perturbation by the base station (BS) which needs to be properly handled on by a UE. Further, as vector perturbation applies a modulo operation at the receiver, we modified the UE processing of the physical downlink shared channel (PDSCH) to perform this operation. In addition to the above, we introduced a pilot boosting scheme. This was necessary to compensate for channel estimation errors (we found that, due to larger transmitted symbol constellation, non-linear approaches are more sensitive to errors in downlink channel estimation). Modifications to the sounding reference signal (SRS) power control to prevent partial loss of channel state information was also introduced.

The hardware components of the SWORD platform are placed in a movable rack to allow for measurements in different locations. As indicated in [13], the main hardware component of the platform is a multi-core workstation with multiple PCle slots which are used for inter-connection with a number of Universal Software Radio Peripheral (USRP) X310 modules. The synchronization and phase alignment across multiple USRPs in SWORD is achieved through the use of Ettus Research Octoclock-G CDA-2990 [30]. The RX and TX paths of each radio chain in the setup are connected via a circulator to an antenna port, which on the BS side is located on an antenna array. On the UE side, antennas are interconnected with their corresponding USRPs via long, low-attenuation cables, and are placed on portable trolleys (see Fig. 2). Note that in the current iteration of the SWORD platform external power amplifiers are not used. This limits the maximum range which in turn restricts the types of scenarios that can be evaluated.

\section{Measurement methodology}

The objective of the measurements is to evaluate the nonlinear beamforming gains compared to linear precoding, in a 3GPP-compliant framework, and to relate the results to the transmission environment. As a metric of the how advantageous the channel is for multi-user transmissions, we chose the channel matrix condition number, and we found that it functions as a useful indicator of the potential gains of non-linear (NL) over linear zero forcing (ZF) precoding.

The condition number of the MIMO channel matrix is a useful metric and provides an indication of how far the MIMO channel is from an orthogonal condition. The condition number of the channel matrix is defined as

$$
\kappa(\mathbf{H})=\frac{\sigma_{\max }(\mathbf{H})}{\sigma_{\min }(\mathbf{H})},
$$

where $\mathbf{H}$ is the channel matrix and $\sigma_{\max }(\mathbf{H})$ and $\sigma_{\min }(\mathbf{H})$ are the largest and smallest singular values of $\mathbf{H}$, respectively.
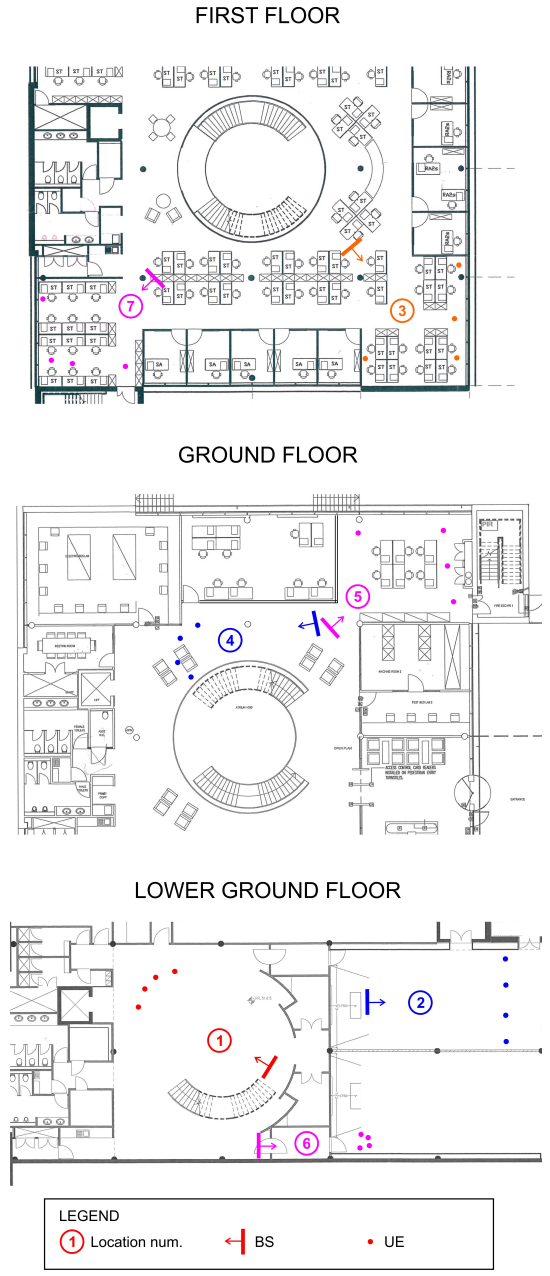

Fig. 1: Floorplan showing the measurement locations.

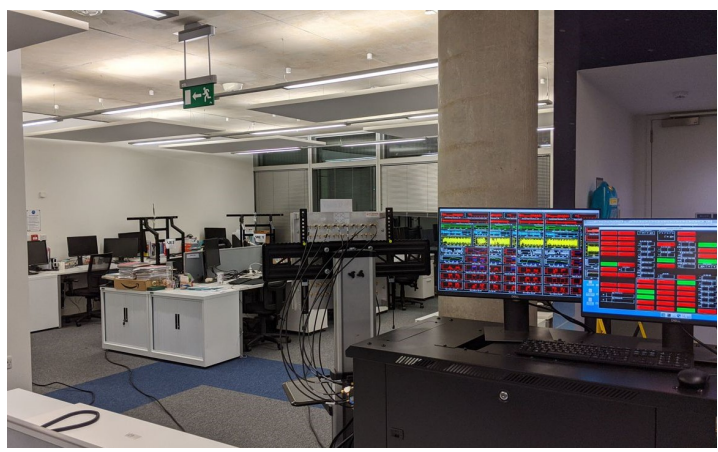

Fig. 2: SWORD setup placement in Location 7.

Smaller values of the condition number are preferred since they indicate that a MIMO channel instance is auspicious for multi-user transmissions and the signal processing needed for successful detection and precoding is small. However, high values reflect an ill-conditioned channel, and in this case linear processing performs poorly, due to the existence of a small singular value [31]. NL techniques are able to improve performance in such situations at the cost of a higher computational overhead. 
In the general sense, MIMO scenarios where the BS has many more antennas than the total number of UE antennas have a low channel matrix condition number, while in cases where there is an equal or a nearly equal number of BS and UE antennas, the condition number is higher [32].

The tests were performed in indoor locations, since these are rich in multipath and provide the opportunity for diverse multiuser environments, due to the existence of multiple reflective, obstructive, and scattering surfaces. Seven indoor locations within 3 floors of the University of Surrey's Institute for Communication Systems building were selected to conduct the measurements; a floorplan of the placement of the BS and UEs is depicted in Fig. 1, with a photograph of one of the locations presented in Fig. 2. The measurements were performed without external power amplifiers (PAs), and due to the limited output power, all locations selected for the indoor measurements had a line-of-sight (LOS) or partially-obstructed LOS path between the BS and each UE. Still, despite the high SNR, the selected locations were demanding due to the linear dependence factor that is introduced from LOS components which make beamforming more challenging when a limited number of BS antennas are used (e.g. overlapping side lobes lead to inter-user interference). All positions were chosen to have distinct characteristics in terms of location and existence of reflection surfaces as well as objects which can scatter the transmitted signal.

As discussed, for NL precoding, the algorithm implemented was the massively parallel non-linear precoder described in [7], with the number of parallel processing paths set to 32 ; this value was selected since it was observed that it provides a good trade-off between error performance and computational complexity. The measurements were conducted for downlink (DL) transmissions in $4 \times 4$ and $8 \times 4$ MU-MIMO scenarios, for linear and non-linear precoding approaches ${ }^{1}$. The platform was set to operate in TDD mode with the LTE DL/uplink (UL) slot configuration number 3 (i.e. 6 DL slots, 3 UL slots and 1 Special Subframe [33]). The carrier frequency selected for the measurement was $3.55 \mathrm{GHz}$, with a channel bandwidth of 5 MHz. The antenna array used for measurements was a $3.4-3.8$ $\mathrm{GHz}$, 128-element rectangular array of dual-polarised $\pm 45^{\circ}$ crossed dipole pairs arranged in an $8 \times 8$ fashion. However, note that only the $+45^{\circ}$ elements of the first row of the array were employed during measurements, making it equivalent to a uniform linear array (ULA). The MU-MIMO scheduler was set such that physical resource block assignment was the same for all the UEs.

Making use of channel reciprocity, the channel estimates at the transmitter were obtained via UL SRS pilots, which were transmitted at every frame. To reduce the effect of thermal noise, in all tests a moving average filter was applied to the SRS channel estimates. Further, to compensate for any phase drift, each measurement instance was preceded by the

\footnotetext{
${ }^{1}$ Note that simultaneous transmission of up to 8 spatial streams is supported by 3GPP LTE, but existing signaling permits only for up to 4 UEs to be scheduled in MU-MIMO transmission.
}

TDD reciprocity calibration procedure. The TDD calibration was followed by a 50-frame warm-up period which was used to determine optimal modulation and coding scheme (MCS), so that the throughput was maximized. A simple algorithm was designed for this purpose based on the tracking of the erroneous and correctly received transmission in DL direction. In addition, in order to reduce the impact of human movement on measurements, the experiments were conducted in confined areas or during night-time. As the platform operated in the "pseudo-RT" mode, this was of particular importance due to a delay between the collection of SRS channel estimates and the exploitation of those measurements for beamforming purposes. Note that in [13] we successfully demonstrated that "pseudoRT" mode can be used to provide accurate performance for beamforming in static MIMO scenarios.

\section{Measurement Results}

This section presents the results and insights obtained from the experiments. The relative gains of the NL precoding technique against the baseline $\mathrm{ZF}$ for $4 \times 4$ and $8 \times 4 \mathrm{MU}-\mathrm{MIMO}$ are presented in Fig. 3 and Fig. 4, respectively. These plots involve the NL throughput gains for each of the tested indoor locations.

The results in Fig. 3 show that in the $4 \times 4$ MIMO case, all the measurement positions exhibit a throughput improvement through the use of NL precoding, with the most gains in Locations 5 and 7 (46\% and 42\%, respectively), and an average gain of approximately $24 \%$. In the case of the $8 \times 4$ MIMO scenario (Fig. 4), a more modest throughput gain is obtained through the use of NL precoding, with an average gain of approximately $4 \%$. The largest gains are obtained in Locations 5 and 6 of approximately $8 \%$, and a very small improvement in Locations 1, 3, and 7. Note that the large difference in the gains between the 4-antenna BS setup and the 8-antenna setup is expected. This is related to the channel matrix which is better conditioned for the $8 \times 4$ case. As discussed in Section IV, when the condition number is low, simple, linear precoding techniques are able to provide good performance, thus limiting the potential benefits of using advanced non-linear methods.

This phenomenon is evident in the condition number of the MU-MIMO scenarios tested. Fig. 5 and Fig. 6 show the empirical cumulative distribution function $(\mathrm{CDF})$ of $\kappa(\mathbf{H})$ for $4 \times 4$ and $8 \times 4 \mathrm{MIMO}$, respectively, for each measurement position tested, where the variation is amongst the orthogonal frequencydivision multiplexing (OFDM) subcarriers. The figures show that the condition number of $8 \times 4 \mathrm{MIMO}$ is lower in average than that of $4 \times 4 \mathrm{MIMO}$; the maximum condition number for the $8 \times 4$ case was approximately 9 , while for the $4 \times 4$ case it was over 40 .

Moreover, within the results for each MIMO size, the NL precoder offers higher throughput gains compared to $\mathrm{ZF}$ for locations with higher condition numbers, in general. This can be observed when comparing the condition number depicted in Fig. 5 and Fig. 6 to that of the throughput gain presented in Fig. 3 and Fig. 4. It is observed that in the locations with a higher condition number (Locations 5 and 7 for the 4-antenna 


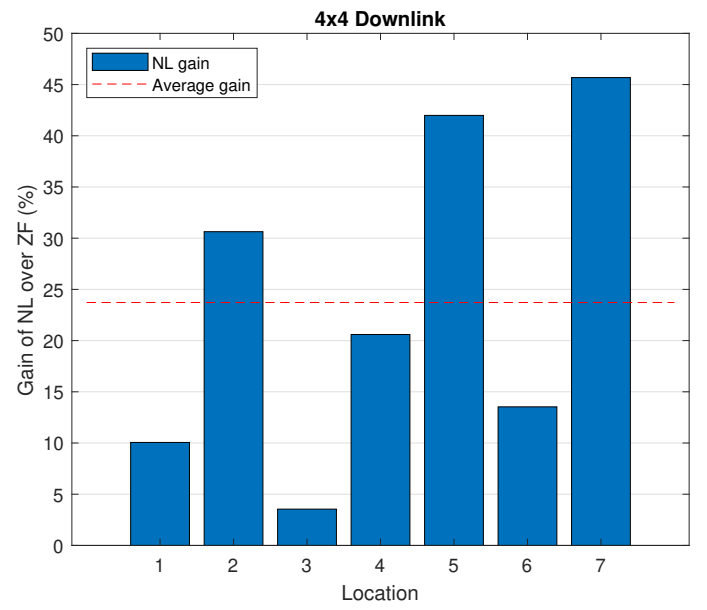

Fig. 3: Relative downlink gain of NL vs. ZF for the $4 \times 4$ MUMIMO configuration in each of the measurement positions.

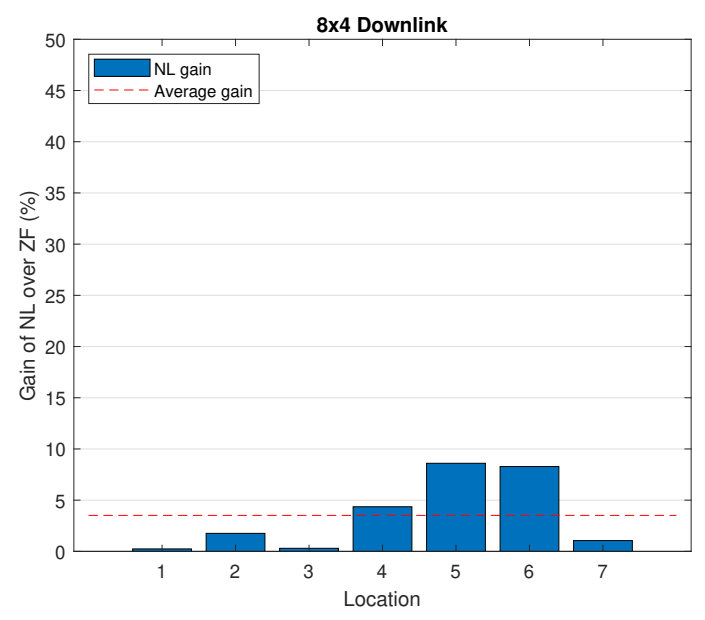

Fig. 4: Relative downlink gain of NL vs. ZF for the $8 \times 4$ MUMIMO configuration in each of the measurement positions.

BS and 4, 5, and 6 for the 8-antenna case) have higher NL gains. However, locations with a higher condition number do not result in throughput gains in all cases (as in Locations 2 and 4 for the $4 \times 4$ MIMO case).

The previous results confirm that the condition number is a good indicator of the potential gains offered by NL precoding, but as a scalar metric, it does not describe the full MIMO environment, hence additional metrics may be needed. Such additional metrics would be helpful to better characterize the MIMO environment and could be used to predict, as an example, the optimal MCS that each data stream / UE should use.

\section{NON-LineAR BEAMFORMing IN 5G}

As discussed in [13], SWORD does not yet fully support the 3GPP 5G New-Radio (NR) standard. However, the non-linear beamforming algorithms are independent of the underlying system and similar extensions would be sufficient for applying them to coming and future mobile standards. In 5G NR the

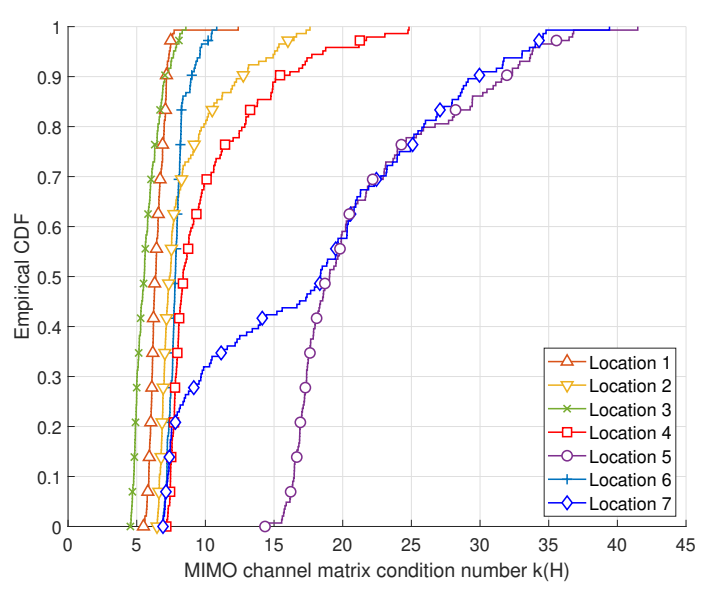

Fig. 5: Empirical CDF of MIMO channel matrix condition number for the $4 \times 4$ MIMO configuration in each of the measurement positions.

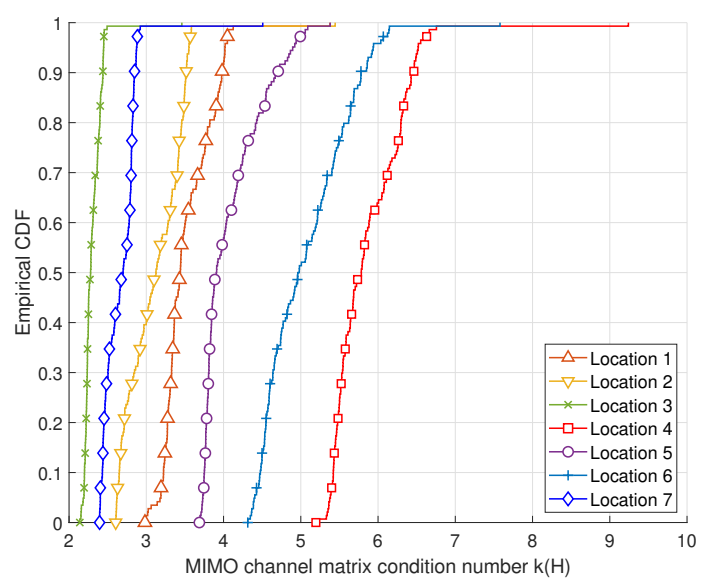

Fig. 6: Empirical CDF of MIMO channel matrix condition number for the $8 \times 4$ MIMO configuration in each of the measurement positions.

gains of non-linear beamforming compared to linear can be even higher than in the case of $4 G$ LTE. This is because the 5G NR standards support a higher number of maximum information streams than $4 \mathrm{G}$ LTE, which are 12 and 8, respectively [33], [34].

\section{CONCLUSION}

In this paper we presented over-the-air, system-level evaluations of the performance of NL beamforming and compared it to linear $(\mathrm{ZF})$ beamforming. For the tests, we made use of SWORD platform, which offers software implementation of a 3GPP standards-compliant protocol stack. By making use of SWORD's "pseudo-RT" mode, we avoided costly and timeconsuming optimizations required for real-time execution of NL precoding algorithms. The measurements were conducted on 7 indoor test locations and for 8- and 4-antenna BS scenarios, while serving 4 single-antenna UEs. The results confirm that the physical-layer gains offered by NL precoding are reflected 
at the system level, with throughput gains of up to $46 \%$ in the case of $4 \times 4$ MIMO when compared to $\mathrm{ZF}$, and gains of nearly $10 \%$ when the number of BS antennas is increased from 4 to 8 . The behavior of the reduction in the gain when the number of BS antennas increases from 4 to 8 is expected. This is confirmed in general by the measurement of the channel matrix condition number obtained from the propagation channels of the test locations. However, as our measurements show, the use of the condition number as a scalar metric does not reflect the complete picture of the MIMO channel, and therefore the use of additional metrics may be necessary to fully describe the gains offered by NL precoding.

\section{ACKNOWLEDGMENTS}

This work has been supported by the UK's Department for Digital, Culture, Media and Sport (DCMS) 5G Testbeds and Trials projects "AutoAir" and "AutoAir II". The Authors would also like to thank the members of the University of Surrey's 5GIC (http://www.surrey.ac.uk/5GIC) for the support as well as Dr G. Georgis, Mr A. Thanos and Dr S. Payami, members of the Wireless Systems Lab of 5GIC, for their contribution.

\section{REFERENCES}

[1] B. M. Hochwald, C. B. Peel, and A. L. Swindlehurst, "A vector-perturbation technique for near-capacity multiantenna multiuser communication-part II: Perturbation,” IEEE Trans. Commun., vol. 53, no. 3, pp. 537-544, 2005.

[2] M. Mohaisen and K. Chang, "Fixed-complexity sphere encoder for multiuser MIMO systems," Journal of Communications and Networks, vol. 13, no. 1, pp. 63-69, 2011.

[3] D. A. Schmidt, M. Joham, and W. Utschick, "Minimum mean square error vector precoding," European Transactions on Telecommunications, vol. 19, no. 3, pp. 219-231, 2008.

[4] C. Yuen and B. M. Hochwald, "How to gain $1.5 \mathrm{~dB}$ in vector precoding," in Proc. IEEE Global Commun. Conf. (GLOBECOM). IEEE, 2006, pp. $1-5$.

[5] H.-S. Han, S.-H. Park, and I. Lee, "Improved vector perturbation with modulo loss reduction for multiuser downlink systems," in Proc. IEEE Int. Conf. Commun. (ICC). IEEE, 2009, pp. 1-5.

[6] W. Yao, S. Chen, and L. Hanzo, "Generalized MBER-based vector precoding design for multiuser transmission," IEEE Trans. Veh. Technol., vol. 60, no. 2, pp. 739-745, 2010.

[7] C. C. M. Husmann and K. Nikitopoulos, "Viper MIMO: Increasing large MIMO efficiency via practical vector-perturbation," Proc. IEEE Global Commun. Conf. (GLOBECOM), 2018.

[8] N. Nikaein, R. Knopp, F. Kaltenberger, L. Gauthier, C. Bonnet, D. Nussbaum, and R. Ghaddab, "Demo: OpenAirInterface: An open LTE network in a PC," in Proc. 20th Annu. Int. Conf. Mobile Comput. and Netw., ser. MobiCom '14. New York, NY, USA: ACM, 2014, pp. 305308. [Online]. Available: http://doi.acm.org/10.1145/2639108.2641745

[9] I. Gomez-Miguelez, A. Garcia-Saavedra, P. D. Sutton, P. Serrano, C. Cano, and D. J. Leith, "srsLTE: An open-source platform for LTE evolution and experimentation," in Proc. 10th ACM Int. Workshop Wireless Netw. Testbeds, Exp. Eval. Characterization, ser. WiNTECH '16. New York, NY, USA: ACM, 2016, pp. 25-32. [Online]. Available: http://doi.acm.org/10.1145/2980159.2980163

[10] openLTE, "OpenLTE; an open source implementation of the 3GPP LTE specifications," https://sourceforge.net/projects/openlte/, 2019.

[11] N. Instruments, "LabVIEW Communications LTE Application Framework 1.1 White Paper," http://www.ni.com/white-paper/52524/en/, 2020.

[12] Amarisoft, "LTE and NR network software suit," https://www.amarisoft. com/products/custom-projects/, 2019.

[13] G. Georgis, M. Filo, A. Thanos, C. Husmann, J. C. De Luna Ducoing, R. Tafazolli, and K. Nikitopoulos, "SWORD: Towards a soft and open radio design for rapid development, profiling, validation and testing," IEEE Access, vol. 7, pp. 186017-186040, 2019.
[14] G. Georgis, A. Thanos, M. Filo, and K. Nikitopoulos, "A DSP acceleration framework for software-defined radios on x86 64," in Proc. IEEE Int. Conf. Acoustics, Speech and Signal Process. (ICASSP), 2020, pp. $1648-1652$.

[15] J. Wang, D. J. Love, and M. D. Zoltowski, "User selection with zeroforcing beamforming achieves the asymptotically optimal sum rate," IEEE Trans. Signal Process., vol. 56, no. 8, pp. 3713-3726, 2008.

[16] T. Yoo and A. Goldsmith, "On the optimality of multiantenna broadcast scheduling using zero-forcing beamforming," J. Sel. Areas Commun., vol. 24 , no. 3, pp. 528-541, 2006.

[17] C. W. Shepard, R. Doost-Mohammady, R. E. Guerra, and L. Zhong, "ArgosV3: An efficient many-antenna platform," in Proc. 23rd Annu. Int. Conf. Mobile Comput. Netw. ACM, 2017, pp. 501-503.

[18] P. Harris, W. B. Hasan, S. Malkowsky, J. Vieira, S. Zhang, M. Beach, L. Liu, E. Mellios, A. Nix, S. Armour et al., "Serving 22 users in realtime with a 128-antenna massive MIMO testbed," in IEEE Int. Workshop Signal Process. Syst. (SiPS). IEEE, 2016, pp. 266-272.

[19] S. Malkowsky, J. Vieira, L. Liu, P. Harris, K. Nieman, N. Kundargi, I. C. Wong, F. Tufvesson, V. Öwall, and O. Edfors, "The world's first realtime testbed for massive MIMO: Design, implementation, and validation," IEEE Access, vol. 5, pp. 9073-9088, 2017.

[20] X. Jiang and F. Kaltenberger, "Demo: An LTE compatible massive MIMO testbed based on OpenAirInterface," in Proc. 21st Int. ITG Workshop Smart Antennas (WSA). VDE, 2017, pp. 1-2.

[21] Z. Qu, "Study to big station management pattern under advanced public transportation systems of China," Database of outstanding master's degree full paper in China, 2007.

[22] M. Costa, "Writing on dirty paper," IEEE Trans. Commun., vol. 29, no. 3, pp. 439-441, 1983.

[23] H. Harashima and H. Miyakawa, "Matched-transmission technique for channels with intersymbol interference," IEEE Trans. Commun., vol. 20, no. 4, pp. 774-780, 1972.

[24] K. Nikitopoulos, "Massively parallel and flexible processing for MIMO systems," Wiley 5G Ref: The Essential 5G Reference Online, pp. 1-21, 2019.

[25] K. Nikitopoulos, G. Georgis, C. Jayawardena, D. Chatzipanagiotis, and R. Tafazolli, "Massively parallel tree search for high-dimensional sphere decoders," IEEE Trans. Parallel Distrib. Syst., 2018.

[26] K. Nikitopoulos and R. Tafazolli, "Parallel processing of sphere decoders and other vector finding approaches using tree search," Aug. 6 2019, US Patent 10,374,841.

[27] C. C. M. Husmann, G. Georgis, K. Nikitopoulos, and K. Jamieson, "Flexcore: Massively parallel and flexible processing for large MIMO access points," in Proc. 14th USENIX Symp. on Netw. Syst. Design and Implementation (NSDI), 2017, pp. 197-211.

[28] K. Nikitopoulos, J. Zhou, B. Congdon, and K. Jamieson, "Geosphere: Consistently turning MIMO capacity into throughput," in Proc. ACM SIGCOMM, vol. 44, 2014, pp. 631-642.

[29] RENEW, "A Reconfigurable Eco-system for Next-generation End-to-end Wireless," https://renew.rice.edu/, 2019.

[30] Ettus, "Octoclock Specification Sheet," https://www.ettus.com/wpcontent/uploads/2019/01/Octoclock_Spec_Sheet.pdf, Jan. 2019.

[31] C. B. Peel, B. M. Hochwald, and A. L. Swindlehurst, "A vector-perturbation technique for near-capacity multiantenna multiuser communication-part I: Channel inversion and regularization," IEEE Trans. Commun., vol. 53, no. 1, pp. 195-202, Jan. 2005.

[32] H. Artes, D. Seethaler, and F. Hlawatsch, "Efficient detection algorithms for MIMO channels: a geometrical approach to approximate ML detection," IEEE Trans. Signal Process., vol. 51, no. 11, pp. 2808-2820, Nov. 2003.

[33] 3GPP, "Evolved Universal Terrestrial Radio Access (E-UTRA); Physical channels and modulation," 3rd Generation Partnership Project (3GPP), Technical Specification (TS) 36.211, Dec. 2019, version 16.0.0.

[34] — "NR; NR and NG-RAN Overall Description; Stage 2," 3rd Generation Partnership Project (3GPP), Technical Specification (TS) 38.300, Dec. 2019, version 16.0.0. 